rev.relac.int.estrateg.segur.10(1):245-265,2015

\title{
EL BANCO MUNDIAL Y LOS DERECHOS HUMANOS: UN REPASO CRÍTICO A LOS ARGUMENTOS JURÍDICO-DOCTRINALES*
}

\author{
Germán Burgos Silva**
}

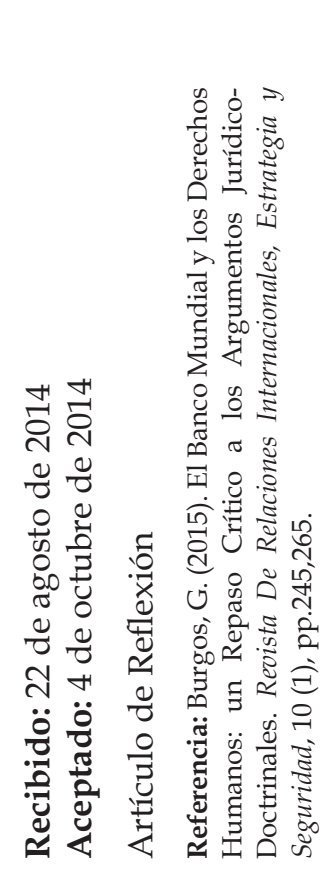

\section{RESUMEN}

Este artículo de reflexión tiene por objeto identificar y valorar críticamente varios de los argumentos jurídico doctrinales que sostienen la obligación jurídica por parte del Banco Mundial de respetar normas internacionales de derechos humanos. Fundado en una metodología cualitativa y centrada en la dogmática del derecho internacional público, sostiene que la vinculación a las Naciones Unidas mediante acuerdo previo, los compromisos internacionales de los estados miembros del organismo y el carácter consuetudinario $y / o$ de principio general del derecho, de esta normatividad, son los tres argumentos centrales de dicha obligatoriedad. Los mismos, sin embargo, están afectados por su generalidad, ambigüedad en cuanto a la

Este es un artículo de reflexión ligado al proyecto de investigación titulado Organismos Económicos Internacionales y Derechos Humanos de la Universidad Libre, Investigación No. 3.

** Profesor de la Universidad Nacional de Colombia y Doctorado en Derecho, Universidad Libre. Abogado de la Universidad Nacional. Doctor en Derecho por la Universidad de Barcelona. Correo electrónico jgburgoss@unal.edu.co 
concreción de los compromisos y no aceptación por el mencionado organismo internacional. En tal sentido, el texto propone la necesidad de promover la suscripción de los tratados de derechos humanos por parte de los organismos internacionales, identificando de paso algunos de los antecedentes ya existentes al respecto.

Palabras clave: Banco Mundial, Organismos Internacionales, Responsabilidad Internacional, Derechos Humanos.

\title{
THE WORLD BANK AND HUMAN RIGHTS: A CRITICAL REVIEW OF THE LEGAL AND DOCTRINAL ARGUMENTS
}

\begin{abstract}
This article aims to critically identify and evaluate a number of doctrinal arguments supporting the legal obligation of the World Bank to respect international human rights standards. Based on a qualitative methodology and focused on the dogmatic of international law, it argues that there are three types of central arguments of this obligation. It can be united in the view that these are general principles of law or international customs binding the International Organizations in general. Likewise, States are committed to these human rights obligations regardless of their membership in international organizations. Finally, the WB is responsible for human rights as a specialized United Nations agency. However all of these statements are affected by their generality, ambiguity and non-acceptance by this international body. In this regard, this document proposes the need to promote the signing of treaties on human rights by international agencies, identifying the current background in this respect.
\end{abstract}

Keywords: World Bank, International Organizations, International Responsibility, Human Rights.

\section{O BANCO MUNDIAL E OS DIREITOS HUMANOS: UMA REVISÃO CRÍTICA A OS ARGUMENTOS JURÍDICO DOCTRINALES}

\section{RESUMO}

Este artigo de reflexão tem por objetivo identificar e apreciar criticamente vários argumentos da doutrina que embasam a obrigação jurídica, por parte do Banco Mundial, de respeitar normas nacionais e internacionais de direitos humanos. Com base em uma metodologia qualitativa e centrada na dogmática do direito internacional público, sustenta que existem três tipos de argumentos centrais da citada obrigatoriedade. Estas são resultantes da sua participação no Nações Unidas por acordo prévio, os compromissos nacionais e internacionais no domínio dos Estados membros do organismo e da natureza habitual e / ou princípio geral de direito internacional de tais compromissos. No entanto, estes, são 
afetados pela generalidade de suas reivindicações, ambiguidade de seus compromissos, e não fácil aceitação pelo mencionado organismo internacional por regulamento interno. Nesse sentido, o texto propõe a necessidade de promover a subscrição dos tratados de direitos humanos por parte dos organismos internacionais, identificando vários antecedentes a esse respeito.

Palavras-chave: Banco Mundial, Organização Internacional, Responsabilidade Internacional, Direitos Humanos.

\section{INTRODUCCIÓN}

El Banco Internacional de Reconstrucción y Fomento (BIRF), ${ }^{1}$ órgano central del conocido grupo del Banco Mundial, continúa siendo la principal fuente multilateral de préstamos y asistencia técnica para los países en desarrollo. ${ }^{2}$ Desde su creación en Bretton Woods, su papel ha crecido a tal punto que hoy se ocupa de múltiples áreas y temas tales como la gobernanza, la reforma a los sistemas judiciales, la modernización del Estado, a más de los típicos proyectos de infraestructura relacionados con carreteras, represas, saneamiento básico, etc. Dado su papel como financiador de importancia para muchos países y su agenda creciente de áreas de interés, entre otros, el BIRF ha sido identificado como un actor clave de la gobernanza global.

El rol central y creciente del BM, ha sido y viene siendo evaluado, entre otros, a la luz de su impacto en materia de DDHH. Efectivamente, desde diversas instancias han surgido inquietudes sobre los alcances que los proyectos y políticas agenciadas por esta organización, han traído para los derechos individuales, sociales y colectivos de distintas poblaciones objeto de su financiación. En particular, las inquietudes al respecto se han planteado en el ámbito de las consecuencias de ciertos proyectos de infraestructura en materia de desplazamiento de personas; en el impacto que tiene el financiamiento de gobiernos autoritarios o con un pésimo record en $\mathrm{DDHH}$; en los efectos cuestionables de los programas de ajuste para los derechos económicos y sociales y finalmente en la forma como los derechos políticos son desconocidos como producto de la condicionalidad (Bank Information Center, 2013) (Clapham, 2006).

En efecto y en primer lugar, el préstamo a gobiernos dictatoriales o comprometidos con violaciones a los DDHH es un tema de vieja data al interior del Banco y tuvo como casos paradigmáticos el financiamiento otorgado al gobierno chileno de Augusto Pinochet y al régimen

1. A lo largo del texto se usarán de manera indistinta expresiones como BM, BIRF, Banco y/o Banco Mundial por razones estilísticas antes que técnicas, como se explicará más adelante. Por su parte, las traducciones del inglés al castellano son responsabilidad del autor.

2. Durante 2013 el BM le prestó a 31 países una suma total de \$9.332.615.273 dólares. Datos obtenidos con base en información presente en la página web del BM. 
sudafricano del Apartheid, aún en contra de las peticiones que para este caso hizo la Asamblea General de la ONU (Brodnig, 2001, p.4). Más recientemente se registró el financiamiento de proyectos en Indonesia bajo el régimen de Suharto y en Zaire bajo la dictadura de Mobutu Sese Seko (Horta, 2002, p.231).

Lo anterior preocupa en la medida en que apoyar financieramente proyectos o programas en países afectados por violaciones a los DDHH, puede terminar por fortalecer a sus gobiernos y sus políticas de conculcación de los mismos, máxime cuando el BIRF no establece ninguna condición para mejorar la situación. Al respecto, la respuesta del Banco ha sido que sin su financiamiento, la situación podría ser peor y que sus estatutos le prohíben valorar la situación política de un país a la hora de aprobar y entregar un préstamo. ${ }^{3}$

Un segundo nivel de impacto en los DDHH es el referente al financiamiento de proyectos de infraestructura y las consecuencias en términos de desplazamiento interno. Según algunas estimaciones, más de dos millones de personas han sido obligadas a dejar sus tierras como producto de grandes proyectos de infraestructura financiados por el BIRF. De hecho, un informe interno del organismo reconoció que de 192 proyectos realizados entre 1986 y 1993, solo uno había compensado adecuadamente a la población afectada por la salida de sus tierras (Clark, 2003) (Gunduz, 2004).

Los efectos de estos reasentamientos parecen darse a nivel especialmente de los derechos económicos y sociales de los afectados y sus familias en cuanto pierden las condiciones para la prestación de ciertos servicios o no pueden afrontar en los mismos términos la satisfacción de sus necesidades. Con todo, en ciertos casos, los derechos civiles y políticos también han resultado conculcados como ocurrió con el proyecto de represa Sardar Sarovar en la India, para cuya realización las autoridades regionales correspondientes, fueron acusadas de intimidaciones y detenciones arbitrarias contra la población que se oponía a esta actividad (Mehta, 2005) (Martin, 2011).

El siguiente ámbito donde los DDHH se han relacionado críticamente con el accionar del Banco, tiene que ver con el impacto de los denominados programas de ajuste estructural en los derechos económicos y sociales de ciertas poblaciones. Luego de la crisis de la deuda, a finales de los 70, el BIRF y otras agencias multilaterales, introducirían el sistema de préstamos por programa, dirigidos a financiar reformas en materia macroeconómica que generalmente impactaban negativamente en el gasto social a cargo de los Estados. En consecuencia, la

3. Recogiendo a Horta, 2002 " According to a writer for the Financial Times, the funding of the regime by western donors, led by the World Bank and the IMF, "locked the society into one slow-motion economic collapse."19 The usual counter-argument made by World Bank staff is that without Bank funding the situation would be even worse" (Horta, 2002, p.231). 
posibilidad de acceder a servicios de salud y educación en un contexto de austeridad se hacía más difícil, impactando negativamente en estos derechos (Adjepoju, 1993). La situación al respecto fue tal, que instancias de Naciones Unidas como el relator para el derecho a la educación, llamaron la atención al respecto:

Para los bancos de desarrollo, la educación figura como parte de los gastos a ser disminuidos a fin de bajar el déficit fiscal. Esto termina entrando en tensión con los requerimientos del derecho internacional en cuanto la distribución de recursos que prioricen el derecho a la educación para permitir que la misma sea libre y obligatoria para todos los niños (Salomon, 2007, p.6). ${ }^{4}$

El cuarto y último ámbito donde los DDHH se han confrontado críticamente con el accionar del BM son los derechos políticos de los ciudadanos(as) de aquellos países objeto de su financiación. En concreto, la llamada condicionalidad atada a los programas de ajuste ha conllevado la introducción de reformas en su política económica y social, desconociendo o neutralizando en la práctica la decisión deliberativa de los órganos de representación popular. Si bien formalmente los congresos adoptan reformas que a primera vista son el producto de su accionar soberano, en el fondo son el producto de la condicionalidad del BIRF. Por esta vía, el alcance de la representación de la población queda vaciado por sustracción de materia. Algo similar ocurre con la asistencia técnica brindada por el BM, la cual según el caso, es la que enmarca la agenda del accionar de ciertas instancias del poder ejecutivo (por ejemplo, Banco Central, Ministerios de Hacienda, entre otros) al margen de la participación de las poblaciones afectadas por las políticas allí generadas (Toussaint, 2007) ( Wahi, 2006).

Planteamientos como los anteriores en torno del accionar del Banco, han tenido entre otros dos inconvenientes. De un lado, algunos de ellos están en el nivel de opiniones genéricas no siempre adecuadamente documentadas y sustentadas con estudios académicos rigurosos. Adicionalmente son planteamientos sujetos a las luchas sociales y políticas en contra de la existencia misma del BIRF, lo cual no está ajeno a ciertos sesgos. En segundo lugar, determinar el nivel de responsabilidad del Banco en la vulneración de los DDHH es un tema complejo dado que a su interior y desde el mismo derecho internacional, no ha sido del todo clara la identificación de cuáles son sus obligaciones concretas en la materia, si es que eventualmente existen (Megnot, 2013).

4. Citado en Salomon, 2007. Recientemente, en 2013, Human Rights Watch (HWR) recogía lo dicho por el Comité de los Derechos del Niño: "[T]he World Bank and other international organizations] should have standards and procedures to assess the risk of harm to children in conjunction with new projects and to take measures to mitigate risks of such harm. These organizations should put in place procedures and mechanisms to identify, address and remedy violations of children's rights in accordance with existing international standards including when they are committed by or result from activities of businesses linked to or funded by them" (HRW, 2013 p.25) 
En este marco y en parte por la presión de diversos actores, el BM ha planteado que la búsqueda del desarrollo, entendido como su objetivo principal, permite avances en DDHH. En igual sentido, ha venido introduciendo por distintas vías la discusión en torno de su papel en relación con la realización de los mismos (Shihatta, 1988).

Por tanto, el objeto central de este texto es identificar qué argumentos jurídicos se han planteado para sostener que el BM tiene obligaciones derivadas de las normas internacionales de DDHH. En otros términos, se pretenden plantear los alcances y las limitaciones conceptuales de los argumentos que desde el derecho internacional se han utilizado para exigir responsabilidades al BM en esta materia.

El método utilizado para el desarrollo de este trabajo es estrictamente cualitativo, basado centralmente en la dogmática del derecho internacional y recoge información de fuentes documentales primarias y secundarias. Desde el punto de vista teórico, el artículo es plural en la medida en que los argumentos aquí recogidos reproducen diversas escuelas dentro del derecho internacional de las Organizaciones Internacionales - OIS.

Para desarrollar lo anterior, el texto está dividido en tres partes. De un lado, ubicaremos brevemente los términos básicos de la subjetividad jurídica del BIRF en cuanto integrante del grupo del Banco Mundial. En un segundo momento presentaremos de manera clasificada cuáles han sido los argumentos jurídicos mediante los que se ha justificado un nivel de obligatoriedad en el respeto a los DDHH por parte del BM y trataremos de determinar que obligaciones surgen al respecto. En una tercera sección haremos un balance transversal de las anteriores argumentaciones a fin de sostener la necesidad de que las OIS suscriban formalmente los tratados de $\mathrm{DDHH}$, como una manera de superar los límites previamente identificados. Al final se incluirá una sección de conclusiones.

\section{EL BANCO MUNDIAL COMO SUJETO DEL DERECHO INTERNACIONAL PÚBLICO (DIP): UNA UBICACIÓN}

El BIRF, institución central del hoy conocido Grupo del Banco Mundial, fue creado en el marco de la conferencia de Bretton Woods bajo los objetivos de soportar financieramente la reconstrucción de Europa luego de la posguerra y suplir las deficiencias de los mercados privados de capital especialmente en lo relativo a la disponibilidad de financiación para el desarrollo. Muy próximo a su creación, la función financiadora del BIRF se vio trastocada como producto de la inauguración del Plan Marshall, el cual se constituyó en el verdadero soporte del proceso de reconstrucción europeo.

Dada esta situación y para comienzos de la década de los años cincuenta del pasado siglo, esta entidad reorientó su labor a la financiación de los países en vías de desarrollo, dentro de los cuales estaban buena parte de los nuevos Estados producto del proceso de descolonización. 
Esta nueva labor de financiación se justificó ante la ausencia de un mercado privado de capitales suficientemente amplio para apoyar las necesidades de dichos países.

Desde hace ya varios años, sin embargo, la financiación al desarrollo no proviene mayoritariamente de la banca multilateral, ya que son los mercados privados los que financian buena parte de los proyectos de desarrollo de forma tal que más del $90 \%$ de esta financiación proviene hoy de bancos privados internacionales antes que del Banco Mundial (Shams, 2004).

Así, el hoy denominado Grupo del Banco Mundial está integrado por cinco instituciones que desarrollan diversos tipos de tareas nunca claramente previstas al momento de su creación. Así, encontramos la Agencia Internacional de Desarrollo, creada en 1960 y encargada de proveer financiación y asistencia técnica a los países con niveles de desarrollo muy bajos. Está igualmente la Corporación Financiera Internacional (CFI), fundada en 1956 la cual realiza lo propio pero respecto del sector privado de la economía. Por su parte, de manera más reciente se han creado el Centro Internacional de Resolución de Disputas en Materia de Inversiones (CIADI) que ofrece servicios de solución de controversias jurídicas en materia de inversiones entre los Estados miembros y la empresa privada, y en los años noventa, se creó la Agencia Multilateral para la Garantía de Inversiones (AMGI), la cual se encarga de proveer seguros a empresas y Estados a fin de proteger a aquellas, de los riesgos políticos que pueden afectar una determinada inversión.

De las cinco organizaciones mencionadas es importante aclarar que cada una de ellas tiene diversas membrecías. Es decir y a manera de ejemplo, los Estados integrantes del BIRF no necesariamente pertenecen al CIADI o a la AMGI y por tanto es dable deducir la existencia de un ámbito de independencia mutua, al menos, formal. Esta es tal que salvo la coordinación entre BIRF y AID, el resto de organismos cuenta con sus propios órganos de dirección y su propio tratado de origen. El presente artículo se dedica fundamentalmente a estos dos últimos.

Al respecto y para poder sustentar la discusión sobre la obligatoriedad para el BIRF de cierta normatividad internacional en materia de DDHH, es importante ubicar brevemente su carácter como sujeto del DIP. En este último, se admite la existencia de las conocidas como organizaciones internacionales o interestatales. Estas son generalmente el producto de un tratado internacional o de la resolución de otra organización previa y el reconocimiento de su subjetividad conlleva la admisión de derechos y obligaciones ante el DIP.

De acuerdo con la doctrina y la jurisprudencia internacional, la subjetividad internacional de una Ol se puede ubicar bajo dos criterios. Uno es su forma de creación, es decir convencional o vía resolución según lo indicado. La otra es la determinación de la existencia de personalidad jurídica o capacidad para asumir derechos y obligaciones. Esta se deduce generalmente de la 
existencia de órganos de dirección diferentes a los de sus Estados integrantes y de las funciones otorgadas a los mismos (Menéndez, 1999) (Espada, 1995).

En este sentido es claro que el BIRF es un sujeto del Derecho Internacional. Así y en primer lugar, fue creado mediante un tratado internacional suscrito en Bretton Woods, que empezó a regir en 1945 y hoy tiene más de 180 Estados miembros. Adicionalmente cuenta con órganos de dirección materializados en la Junta de Gobernadores que se reúne una vez al año y está integrada por representantes de los Estados miembros. Adicionalmente está el Consejo de Dirección, instancia colectiva de 24 personas que representan a los integrantes de la Junta y que es encargada de tomar las decisiones rutinarias de la organización. Entre las atribuciones de la Junta están, celebrar acuerdos con organizaciones internacionales. En igual sentido, el tratado que lo conforma establece en su art. 7, sección 2, que El Banco deberá tener plena personalidad jurídica y, en particular, la capacidad de: i) celebrar contratos; ii) adquirir bienes muebles e inmuebles y disponer de ellos y entablar acciones judiciales.

En suma, el Grupo del Banco Mundial, está integrado de manera central por el BIRF, organización internacional de especial importancia dado su papel como financiador y ofertante de asistencia técnica para el desarrollo. Este constituye un sujeto del DIP en la medida en que ha sido conformado a través de un tratado y se le reconoce personalidad jurídica expresada en sus órganos de dirección. En cuanto sujeto del derecho internacional es por tanto posible preguntarse qué derechos y obligaciones existen para el BIRF en materia del derecho internacional de los DDHH.

\section{EL BANCO MUNDIAL Y SUS OBLIGACIONES EN DERECHOS HUMANOS}

Es creciente el número de actores estatales, sociales y académicos que han venido postulando diversos argumentos para comprometer al Banco Mundial en el respeto y protección de los DDHH reconocidos nacional e internacionalmente (Agusti \& Panareda, 2008) (Ciorciari, 2000) (Ghazi, 2005) (Mcbeth, 2008) (McCorquodale, 2009). Según un estudio del mismo BM en el marco de la revisión de sus políticas de salvaguardia, parte de dicho interés parece centrarse especialmente en los gobiernos de algunos países desarrollados y en representantes de diversos actores sociales nacionales y/o globales, mientras los gobiernos de los países prestamistas aparecen como muy refractarios frente al tema. ${ }^{5}$

Con todo, las directivas del Banco han sido muy reticentes en aceptar responsabilidades en la materia apelando fundamentalmente a su apoliticidad, prevista en su tratado de

5. En efecto, en una encuesta hecha a participantes de las discusiones arriba anotadas, quedó claro que para casi un $90 \%$ de los donantes y un 50\% de los actores de la sociedad civil, el tema era prioritario, mientras menos de un $9 \%$ de los prestamistas lo consideraban como tal ( Banco Mundial, 2013, p.25). 
conformación, la cual por lo demás según algunos se sustenta en un derecho humano de carácter colectivo como es la autodeterminación de los pueblos. Así las cosas, el BM no podría intervenir en los temas de DDHH al ser temas políticos propios de la autodeterminación nacional. ${ }^{6}$

A pesar de las resistencias desde el BM, son varios los argumentos jurídicos que se han esgrimido a fin de obligar a aquel a asumir obligaciones en términos de los DDHH reconocidos internacionalmente. Los mismos se pueden clasificar en tres. El primero de ellos hace referencia a las obligaciones internacionales en DDHH de los Estados miembros del BM y el impacto sobre este. El segundo tipo tiene que ver con la relación especial entre el BIRF y las Naciones Unidas. Por último, están aquellas tesis que consideran que existen razones jurídicas de peso para considerar que el derecho internacional de los DDHH es una fuente en si misma vinculante para el Banco.

A continuación desarrollaremos este grupo de tesis, tratando de ubicar sus fundamentos y límites de diverso tipo. Luego de esto, haremos una lectura crítica transversal sobre su alcance real para defender la idea de abrir paso a la suscripción de los tratados de derechos humanos por las OIS, incluido el BM, como por distintas vías se ha venido haciendo.

\section{EL BANCO MUNDIAL Y LAS CONSECUENCIAS DE SU VÍNCULO CON LAS NACIONES UNIDAS}

Desde 1947 y mediante un tratado internacional, el BM al igual que el FMI, se entienden como organismos especializados de las Naciones Unidas. Dada esta relación se ha sostenido que el BM está sujeto a la Carta de esta organización, la cual incluye dentro de sus objetivos básicos el "desarrollo y estímulo del respeto a los derechos humanos y a las libertades fundamentales de todos, sin hacer distinción por motivos de raza, sexo, idioma o religión". Por esta vía, dicho organismo debería estar comprometido al menos con el respeto y desarrollo de los DDHH. Por lo demás, según Skogly, 2001, esto incluiría una obligación de neutralidad entendida como no agravar la situación de DDHH en un determinado país. ${ }^{7}$

6. Retomando a Salomón "The prohibition was, in a sense, designed to protect a human right, the right to selfdetermination: the right to choose one's economic and political system. Such a prohibition can hardly serve to create a general impunity from human rights accountability for the international organization" (Salomón, 2007, p.13).

7. Según Gunduz "However, the protection of human rights is one of the central principles and functions of the UN, as laid down in the UN Charter (Article 1(3)). While the Bank is legally and organisationally independent from the UN, it is not independent to the degree of violating fundamental UN principles, but has to respect them. Inasmuch as the International Bill of Human Rights elaborates and codifies the UN Charter's human rights principle (see section 1.1), the Bank's obligation" (Gunduz, 2004, p.25). 
Adicionalmente, la Carta de la ONU establece en su artículo 103 que en caso de conflicto entre las obligaciones contraídas por los Miembros de las Naciones Unidas y aquellas contraídas en virtud de cualquier otro convenio internacional, prevalecerán las obligaciones impuestas por esta última. En otros términos, si el Banco Mundial forma parte de la ONU, el tratado básico de esta debe considerarse superior al convenio mediante el cual se creó aquel. Por tanto, el BM estaría obligado a respetar las obligaciones básicas en DDHH que surgen de la Carta de la ONU, no solo al ser un órgano especializado del organismo sino dada la superioridad jerárquica proveniente del art. 103.

Esta argumentación, sin embargo, ha tenido dos inconvenientes. El primero ha sido la reticencia histórica dentro del BIRF a asumir de manera plena su carácter como organismo especializado de la ONU, aduciendo que este vínculo es ante todo de coordinación pero que no conlleva subordinación jerárquica ni a la Asamblea General ni al tratado conformador de aquella. El BM siempre ha defendido que su relación con la ONU no puede poner en tela de juicio su autonomía e independencia (Toussaint, 2005). Lo máximo que ha aceptado es obedecer resoluciones vinculantes del Consejo de Seguridad en desarrollo de lo previsto en capítulo 7 de la Carta (Brown, 1992, p.132).

De otra parte, aún en el caso de que se aceptara este argumento, lo que no queda claro por ahora es, cuáles serían tales derechos y si podrían finalmente homologarse a los distintos tratados que se han negociado en el marco de las Naciones Unidas. En igual sentido, no se ha determinado aún que tipo de obligaciones concretas generaría esta normatividad para el BM (Koskenniemi, 2007).

\section{LAS OBLIGACIONES INTERNACIONALES DE LOS ESTADOS Y SU VÍNCULO CON EL BANCO MUNDIAL}

Bajo esta argumentación se sostiene que las obligaciones de los Estados respecto de los tratados de $\mathrm{DDHH}$ por ellos ratificados, no se suspenden por pertenecer a ciertas organizaciones internacionales como los organismos multilaterales de crédito y que por tanto sus deberes en materia de derechos deben compatibilizarse con su accionar, en este caso, dentro del BM (Ryngaert y Buchanan, 2011) (Martín, 2008).

Como se ha afirmado de tiempo atrás, buena parte de los Estados que integran al Banco, han ratificado a su vez varios tratados de $\mathrm{DDHH}$, incluidos los más icónicos como el pacto de derechos civiles y políticos y el de derechos económicos y sociales. Efectivamente, más del 90\% de los 188 integrantes del BM han ratificado cuatro o más tratados de DDHH. A manera de ejemplo, 181 lo han hecho con la Convención contra toda forma de Discriminación contra la Mujer; 170 hicieron lo propio respecto del Convenio Internacional sobre Derechos Civiles y políticos, mientras 170 tienen obligaciones con el Pacto de Derechos Económicos y Sociales (Human Rights Watch, 2013, p.16). 
De lo anterior se colige que la pertenencia a una determinada organización internacional no puede entenderse como una forma de eludir sus compromisos en DDHH y por el contrario siguen sujetos a ellos. De hecho, en cuanto los Estados tienen obligaciones en la materia, deben tratar de cumplirlas, entre otros, por medio de las organizaciones internacionales de las que hacen parte. ${ }^{8}$

Bajo la anterior argumentación, el Comité del Pacto de Derechos Económicos y Sociales ha planteado que los Estados de los países desarrollados, deben "como miembros de las IFIs, "hacer todo para asegurar que las políticas y decisiones del Banco Mundial y el FMI, estén de acuerdo con las obligaciones de los Estados partes respecto de la Convención" (Salomon, 2007, p.134).

Un claro fundamento de lo anterior, sería el artículo 31.3C de la Convención de Viena de los Tratados. En esta se afirma, que un tratado deberá interpretarse de buena fe conforme al sentido corriente que haya de atribuirse a sus términos en el contexto de estos y teniendo en cuenta su objeto y fin. Adicionalmente en la interpretación no solo se debe tener en cuenta el texto del tratado sino "toda forma pertinente de derecho internacional aplicable en las relaciones entre las partes".

En otros términos, el tratado constitutivo del BM y las funciones y roles que allí se establecen, no solo puede mirarse a la luz de su texto, por más claro que este sea. Complementariamente deben tenerse en cuenta otras expresiones del derecho internacional aplicable en las relaciones entre las partes, lo cual incluiría los tratados de DDHH suscritos por los países que a su vez integran esta organización. Para interpretar el tratado original del BM, por tanto, se deben tener en cuenta los tratados de DDHH vigentes igualmente entre sus países miembros. ${ }^{9}$

Haciendo eco en lo anterior, algunos doctrinantes del derecho internacional han realizado importantes desarrollos a través de los principios de Maastricht sobre las obligaciones extraterritoriales de los Estados en el área de los derechos económicos, sociales y culturales y también por medio del Informe de expertos sobre los efectos de la deuda externa y otras

8. Según Wahi "There are others who argue that States that are members of these institutions and are also party to the various human rights treaties have an obligation to seek implementation of these treaties not only in their bilateral relations with other parties, but also through their actions in international organizations. This in turn imposes an indirect obligation upon the World Bank and the IMF to comply with human rights norms while implementing their policies and projects (Wahi, 2006, p.335).

9. Según Lindros "It would have been of particular interest to test Article $31(3)(\mathrm{c})$ as a potential tool to allow the mandates to be interpreted in the light of human right law as it stands today. This paragraph allows an incorporation of legal rules outside the agreement itself, and perhaps makes it possible to take human rights treaties which the institutions are not party to into account. Of course, this would also have raised the difficult question of the intertermporal aspect of treaty interpretation" (Lindros, 2006, p.1041). 
obligaciones financieras internacionales respecto del disfrute de los DDHH. Este último plantea de manera categórica que:

Todos los estados, actuando individual o colectivamente, aún como integrantes de organizaciones internacionales, tienen la obligación de respetar, proteger y cumplir los derechos humanos. Es así como deben asegurar que todas y cada una de las actividades relativas a las decisiones de préstamos, la utilización de fondos, la reestructuración de la deuda externa... no deben derogar tales obligaciones (HRW, 2013, p.28).

A pesar de los anteriores fundamentos, son dos los límites de los mismos. El primero remite al poco interés de los Estados por colocar expresamente el tema de las obligaciones internacionales de estos al interior del Banco y sus órganos directivos. De manera algo sorprendente, ha sido la representación británica quien ha intentado algo al respecto (Salomón 2007). Por su parte, Estados Unidos cuenta con una legislación interna que obliga a su gobierno a tener en cuenta la situación de DDHH a la hora de aprobar un préstamo, pero el uso de esta facultad no está atado a los compromisos internacionales de este país sino a la política de su gobierno, la cual suele estar sujeta a diversos tipo de intereses (Gunduz, 2004). De otro lado, como se planteó con antelación, el interés de los gobiernos de los Estados prestamistas, parecer ser muy bajo para colocar el tema al interior de la organización.

Finalmente, desde algunos sectores de especialistas se ha planteado que las referencias a la Convención de Viena arriba anotadas son impertinentes en cuanto los tratados de DDHH no refieren como tal a las relaciones entre Estados sino a la de estos con sus poblaciones. Siendo esto cierto no se puede negar que los tratados de DDHH vinculan a los Estados respecto de otros en cuanto existe un interés internacional por su avance permanente, lo cual conlleva el monitoreo mutuo entre estos y obviamente el apoyo de determinados organismos internacionales para promover su cumplimiento.

\section{LAS NORMAS INTERNACIONALES DE DERECHOS HUMANOS COMO FUENTE VINCULANTE PARA EL BANCO MUNDIAL}

Un importante sector de los interesados en este tema ha planteado que el BIRF estaría obligado a respetar normas de DDHH internacionalmente reconocidas en cuanto estas son vinculantes a título de costumbre internacional o principio general del derecho.

En efecto y en primer lugar, se sostiene que las normas de DDHH corresponden a principios básicos del derecho internacional. Para ello se fundamentan en el artículo 38 del Estatuto de la Corte Internacional de Justicia, según el cual constituyen una fuente del derecho los principios generales aceptados por las naciones civilizadas del mundo de forma tal que por esta vía, los DDHH harían referencia a este tipo de principios. Dado que el BM no ha suscrito tratados 
en la materia, estaría obligado a respetar normas atinentes al respecto en cuanto constituyen principios generales del derecho. ${ }^{10}$

En un sentido más general y aceptado está el fundamento consuetudinario de las obligaciones de $\mathrm{DDHH}$ del BM. Al respecto es claro que los tratados sobre el tema son por lo general discutidos, negociados y ratificados por Estados y no por OIS. Con todo, respecto de los demás actores del derecho internacional, las prácticas generadas a raíz de las normas internacionales de DDHH, conforman una obligación jurídica a título de costumbre. En otros términos, las OIS según el ámbito de sus funciones y su mandato deben respetar un catálogo de DDHH en cuanto costumbre internacional. Al respecto, la Corte Internacional de Justicia en el caso WHO, dejó claro que aquellas están "limitadas por cualquier obligación sobre la base del derecho internacional consuetudinario". ${ }^{11}$

En este contexto, uno de los puntos en cuestión hace referencia a qué DDHH formarían parte del anterior catálogo. Una primera respuesta sostiene que claramente el Banco está obligado a respetar los derechos cobijados bajo las normas de jus cogems o imperativas internacionales. Así, por encima de su declarada apoliticidad, recogida en su tratado constitutivo, estas últimas constituyen mandatos superiores que deben ser respetados obligatoriamente por todos los sujetos del derecho internacional.

Más allá de lo anterior, autores como De Feyter, 2002 y Rosas, 2007, han planteado que dentro del derecho consuetudinario aplicable, estaría la Declaración Universal de los Derechos Humanos. Esta tiene hoy por hoy estatus de costumbre vinculante hasta para los mismos Estados dado que formalmente no constituye un tratado como tal. Por su parte, el alcance de la Declaración parece tener una especial fuerza respecto del Banco dado el contenido de algunas de sus normas. En particular, se ha resaltado como los artículos 25 y 28 son suficientemente específicos respecto del accionar de este:

Art. 25. 1 Toda persona tiene derecho a un nivel de vida adecuado que le asegure, así como a su familia, la salud y el bienestar, y en especial la alimentación, el vestido, la vivienda, la asistencia médica y los servicios sociales necesarios; tiene asimismo derecho a los seguros en caso de desempleo, enfermedad, invalidez, viudez, vejez u otros caso de pérdida de su medios de subsistencia por circunstancias independientes de su voluntad.

10. Recogiendo a Wouters y otros, "Other authors consider that human rights have become general principles of international law through the medium of the 'general principles of law recognized by civilized nations' mentioned in Article 38(1) (c) of the Statute of the international Court of Justice.29 Such general principles would not require" (Wouters, Brems y Smis y Schmitt, 2010, p 7 ).

11. Según, Suzuki, "International organizations are, as stated by the ICJ in the WHO case, "bound by any obligations incumbent upon them" under customary international law" (Suzuki, 2006, p.190). 
Art. 28. Toda persona tiene derecho a que se establezca un orden social e internacional en el que los derechos y libertades proclamados en esta Declaración se hagan plenamente efectivos.

Adicionalmente, existen planteamientos que sostienen que la costumbre internacional incorpora no solo las normas de jus cogems y la Declaración, sino buena parte de los tratados internacionales de DDHH. En tal sentido, vía la costumbre, el BM debería enmarcar su accionar en el respeto, protección y promoción de los derechos integrados a buena parte de los tratados (Días, 2013).

La anterior argumentación, sin embargo, estaría afectada por varias dificultades. De un lado, no es fácil determinar qué normas de DDHH forman parte como tal de la costumbre internacional, más allá de aquellas imperativas cuyo alcance aparece como limitado para el Banco. Formalmente en 2015 no existe una organización internacional que declare de manera precisa cuáles serían este tipo de normas. Por lo demás, los derechos económicos y sociales, especialmente relacionados con el accionar del Banco, casi nunca han sido considerados a título de costumbre. Por tanto, no es posible determinar fácilmente sobre que normas se podrían generar compromisos específicos para el BM. ${ }^{12}$

De otra parte, existe un debate irresuelto sobre sí organismos como el BIRF por su estructura, podrían tener responsabilidades en la materia. En concreto, se sostiene que por su tipo de accionar expresado en asistencia técnica y préstamos, este no tendría relación directa con las poblaciones y por tanto en su ámbito de acción no tiene posibilidad de afectar los DDHH, salvo de manera muy indirecta. Por tanto, en el eventual caso de estar sometido a la costumbre internacional, no sería fácil determinar en qué términos sería dado lo anterior. ${ }^{13}$ Adicionalmente, una de las normas aceptadas por el derecho internacional, tiene que ver con la imposibilidad de los actos ultravires. Es decir, las OIS, entre otros, solo pueden actuar en el marco de sus funciones y atribuciones, las cuales, en el caso en mención no incluirían obligaciones en materia de $\mathrm{DDHH}^{14}$ Por tanto, sería en principio difícil que el Banco asumiera compromisos que inicialmente no serían claros frente a su tratado constitutivo.

12. Según De Feyter, 2002 "A clear disadvantage of having to rely on custom and general principles is that it opens up the space for challenges to the status of the rule, if only because there is no standing mechanism with the authority to review and determine whether specific human rights obligations have achieved the necessary status or not" (De Feyter, 2002, p.9).

13. Contrariamente a esta posición, Wahi considera que "the World Bank and the IMF. My claim is that that these institutions are exercising "governing" power directly over individuals within their member states. The use of such governing power justifies direct human rights accountability of these institutions" (Wahi, 2005, p.334).

14. "Consequently, the degree to which the international financial institutions are bound by affirmative duties to act10 towards the realization of general rules of human rights needs to be determined in the light of the constituent documents and subsequent practice of the organizations. The World Bank and the IMF can only be required to engage in activities for the realization of human rights to the extent allowed by their respective purposes and functions" (De Feyter, 2002, p.8). 


\section{UN CAMINO HACIA LA INCORPORACION DE LOS TRATADOS DE DERECHOS HUMANOS POR LAS OIS}

Como puede verse, hay un amplio tipo de argumentos jurídicos que especialmente la doctrina ha planteado para establecer obligaciones en DDHH a organismos como el BM. Con todo, dichas tesis están afectadas por diversos tipos de críticas que tienen que ver con vacíos en la fundamentación, escasa apropiación por los actores o excesiva ambigüedad en torno de los compromisos concretos que debería asumir esta organización.

Con todo, hay una limitación transversal a todas las argumentaciones arriba identificadas. A pesar de los debates doctrinales, hoy no existe un mecanismo institucional, ni internacional ni nacional que haya logrado de manera sistemática, conocer y juzgar el comportamiento de las OIS en materia de DDHH. Las OIS por lo general, no le han dado competencia a ningún organismo para el efecto y los Estados integrantes o no de las mismas, no han presionado claramente para que esto exista. En un contexto donde los Estados están cada vez más sujetos a OIS de diversos tipos, éstos a su vez parecen no aceptar formas de control y juzgamiento externos de carácter institucional (Rey, 2006)..$^{15}$

Las dificultades de juzgamiento internacional, se repiten a nivel nacional. Dado el principio de inmunidad de jurisdicción, reconocido de forma amplia a buena parte de las OIS, estas no pueden ser demandadas de forma viable en los tribunales internos de los países con los que tienen algún nivel de relación formal (Young, (2012). En tal sentido, no es posible demandar a un organismo como el BM, en los tribunales de un Estado cuya población se haya visto afectada por el accionar del organismo. De hecho, el tratado constitutivo de aquel prevé la posibilidad de demandar a nivel judicial nacional solo por asuntos relacionados con los títulos de deuda emitidos por este pero no respecto de otros temas. ${ }^{16}$

A pesar de la inmunidad de jurisdicción, los tribunales nacionales han sido una de las pocas vías para tratar de obtener responsabilidad de las OIS, especialmente en casos que han tenido que ver con derechos laborales de sus trabajadores. Esto al menos ha sido la experiencia en los Estados Unidos frente a casos relacionados con el accionar de la ONU. En concreto, el más reciente versa sobre la demanda que algunos ciudadanos haitianos presentaron en 2013 contra este organismo en algunos tribunales federales de la ciudad de Nueva York. La misma hace referencia a las muertes asociadas a la transmisión del cólera en territorio haitiano, por parte de los cascos azules, en particular los de origen nepalí. Hasta el momento, la ONU se ha

15. Una situación registrable aquí es el caso del Tribunal Europeo de Derechos Humanos en el caso Behrami y Saramati donde a pesar de no haber asumido competencia, planteó que las muertes objeto del litigio no eran asignables a los estados que participaron en Kosovo como parte de la ONU, sino a este organismo y en particular al Consejo de Seguridad. Lo relevante de este caso es que un organismo como el Tribunal en mención, establezca que la responsabilidad es de una OI aunque por ello no puede asumir competencia (Larsen, 2008).

16. Sólo podrá seguirse acción contra el Banco ante un tribunal de jurisdicción competente, en los territorios de un miembro donde el Banco tuviere establecida una oficina, en que hubiere designado un apoderado con el objeto de aceptar emplazamiento o notificación de demanda judicial, o donde hubiere emitido o garantizado títulos (art. 7, 3). 
negado a asumir responsabilidad alguna y la vía más clara, aunque compleja, encontrada por las víctimas, han sido instancias judiciales estadounidenses. ${ }^{17}$

En el anterior contexto de vacío de institucionalidad internacional y nacional, una vía que se ha venido explorando, es presionar por diversos medios la incorporación expresa, mediante diversas fórmulas, de las normas internacionales de DDHH por las diversas OIS. En otros términos para dar un salto cualitativo respecto de esta discusión, parece más que oportuno profundizar en la agenda internacional en torno a que las OIS se comprometan clara y expresamente con un conjunto de normas internacionales en la materia.

Al respecto hay varias vías. Una de ellas sería la incorporación unilateral de criterios de accionar basados en las normas de DDHH por parte de las OIS. Esto significaría tener una política interna en la materia, basada en la normatividad internacional. La otra fórmula es la suscripción formal de los tratados de DDHH. Es decir, promover y permitir que las OIS puedan vincularse oficialmente a los compromisos internacionales en la materia.

A partir de estos modelos, se ha venido dando un nivel de involucramiento progresivo y diferencial de diversas OIS en relación con sus obligaciones en materia de DDHH. Por ejemplo, el Banco Europeo de Inversiones y su homólogo de Reconstrucción y Desarrollo, se han comprometido a no financiar proyectos que puedan resultar en la violación de DDHH. Por su parte, el Banco Asiático de Desarrollo ha dejado claro que los tratados internacionales de diversos tipos deben respetarse a la hora de suscribir acuerdos de préstamo. Adicionalmente las 32 organizaciones que integran a la ONU, se han comprometido a tener una aproximación de DDHH al desarrollo según la cual este debe propender por la realización de aquellos en los términos previstos en la Declaración Universal y en los demás instrumentos internacionales al respecto (Human Rights Watch, 2013) (OCDE, 2013). La mismísima OTAN y en particular la Misión que administraba provisional y parcialmente Kosovo, aceptaron unilateralmente ciertas normas de $\mathrm{DDHH}^{18}$. Finalmente, de una forma más categórica, la Unión Europea ha suscrito la Convención contra la discapacidad de la Unión Europea. ${ }^{19}$

17. Según cita De Feyter, "Dominicé has argued that the jurisdictional immunity of international organizations before domestic courts should not prevail over the human rights of private individuals adversely affected, particularly if the individual does not have access to any other tribunal" (De Feyter, 2002, p.22).

18. "This has been the case for UNMIK and the North Atlantic Treaty Organisation (NATO) operating in Kosovo. In addition to having unilaterally accepted to be bound by the provisions of a number of human rights treaties, they also submitted to monitoring procedures. The first such act was the conclusion of an agreement between UNMIK and the Council of Europe in relation to the Framework Convention for the Protection of National Minorities (ETS No. 157) in 2004. This requires UNMIK to submit reports to the Committee of Ministers, which may address recommendations to UNMIK. Similar agreements are in place regarding visits of the European Committee for the Prevention of Torture and Inhuman or Degrading Treatment or Punishment (CPT) to places where persons are deprived of their liberty in Kosovo by UNMIK and NATO" (Consejo de Europa, 2013).

19. Como bien recoge Salomon, "As De Schutter has rightly remarked, though, it would be a faulty approach to have as a condition of joining a human rights treaty that the organization has competence in the area of human rights, as is the case for example with regard to the European Community and the recently adopted UN Convention on Persons with Disabilities" (Salomon, 2007, p.21). 
El mismo BIRF desde hace algún tiempo, ha venido tomando las que se conocen como políticas de salvaguardia a nivel social, ambiental y de reasentamientos. Si bien las mismas no asumen como tal un lenguaje de DDHH ni compromisos basados en el derecho internacional, se consideran un avance en el anterior sentido. De hecho, esta investigación, pretende continuar tratando de ubicar el contenido y el alcance de estas políticas desde el BM.

\section{CONCLUSIÓN}

El presente texto ubicó y valoró críticamente cuáles han sido los argumentos jurídico doctrinales más reiterados en favor de la obligación del BM de respetar normas internacionales de DDHH. Su especial aporte radica en poner en conjunto buena parte de los argumentos esgrimidos, identificando su contenido central y evaluando sus alcances y limitaciones.

En tal sentido, se concluye que hay al menos tres tipos de argumentos. De un lado el BM en cuanto parte de la ONU, estaría obligado a respetar su tratado constitutivo, el cual expresamente hace referencia a los DDHH. En segundo lugar está la invocación del artículo 31 de la Convención de Viena, el cual a su vez complementa la tesis según la cual los compromisos de los Estados en materia de DDHH los siguen aún bajo su pertenencia a Organizaciones Internacionales.

Finalmente, está la tesis según la cual los DDHH son principios generales de derecho y/o costumbre internacional vinculantes para las OIS en general. Las anteriores han sido cuestionadas por su poca operatividad, por su limitada fundamentación o escasa eficacia.

Salvo las normas de jus cogems, cuyo contenido no es del todo claro aparte de situaciones extremas, no es fácil determinar, a partir de los anteriores argumentos, que normas de DDHH serían oponibles al Banco respecto de su accionar. Doctrinalmente hay un debate irresuelto al respecto y no hay muchos avances aclaratorios.

Lo mismo ocurre con la identificación del tipo de deberes y el contenido de los mismos, en cabeza del BM. En otros términos, aun conociendo las normas que lo vinculan, tampoco es claro cuáles serían las obligaciones concretas del organismo según de que derecho humano estemos hablando.

Dado el anterior contexto, una opción que se ha venido abriendo de diversas formas ha sido la incorporación de normas de derechos humanos por las Ols como tal. En tal sentido, esto se puede hacer mediante la generación de políticas internas que adapten unilateralmente la normatividad internacional al respecto o mediante la suscripción de los tratados correspondientes por las IFIS.

Otra vía es la generación de políticas internas desde la organización internacional, aunque sin tener en cuenta de manera directa la normatividad existente. Este parece que ha sido el camino seguido por el BM. 


\section{REFERENCIAS}

Agusti-Panareda, J. (2008). Transforming Weakness into Virtue-A Dialogue and Context Based Approach to Human Rights at the World Bank. Hum. Rts. \& Globalization L. Rev., 2, 23.

Adepoju, A. (1993). The impact of structural adjustment on the population of Africa: the implications for education health and employment.Nueva York. UNFPA.

Brown, B. S. (1992). The United States and the politicization of the World Bank: issues of international law and policy. Routledge.

Burgos J. G. (2009). Estado de derecho y globalización: el papel del Banco Mundial y las reformas institucionales en América Latina. Universidad Nacional de Colombia, Facultad de Derecho, Ciencias Políticas y Sociales, Departamento de Ciencias Políticas.

Ciorciari, J. D. (2000). Lawful Scope of Human Rights Criteria in World Bank Credit Decisions: An Interpretive Analysis of the IBRD and IDA Articles of Agreement, The. Cornell Int'I LJ, 33, 331 .

Clark, D. L. (2002). World Bank and Human Rights: The Need for Greater Accountability, The. Harv. Hum. Rts. J., 15, 205.

Clapham, A. (2006). Human right obligations of non-state actors. Londres: Oxford University Press.

De Feyter, K. (2004). The international financial institutions and human rights: Law and practice. Human Rights Review, 6(1), 56-90.

Dias, L. A. (2013). La tendencia hacia un rol fortalecido de las normas de" Jus Cogens" en el Derecho Internacional: caminos, riesgos y utopías. Revista Electrónica del Instituto de Investigaciones Jurídicas y Sociales Ambrosio Lucas Gioja, 7(10), 45-70.

Espada, C. G. (1995). Derecho internacional público. Madrid:Trotta.

Ghazi, B. (2005). The IMF, the World Bank group and the question of human rights. Nueva York: Transnational Publishers.

Herbertson, K., Thompson, K., \& Goodland, R. J. (2010). A roadmap for integrating human rights into the World Bank Group. Washington, DC: World Resources Institute.

Horta, K. (2002). Rhetoric and Reality: Human Rights and the World Bank.Harv. Hum. Rts. J., 15, 227. 
Larsen, K. M. (2008). Attribution of conduct in peace operations: the 'Ultimate Authority and Control'test. European Journal of International Law, 19(3), 509-531.

Lindros, A. Books Reviews. EJIL 17 (2006), 1033-1047.

Martín, J. M. C. (2008). Las organizaciones internacionales: codificación y desarrollo progresivo de su responsabilidad internacional. Instituto Andaluz de Administración Pública.

McBeth, A. (2008). Right by Any Other Name: The Evasive Engagement of International Financial Institutions with Human Rights, A. Geo. Wash. Int'l L. Rev., 40, 1101.

McCorquodale, R. (2009). International Organizations and international human rights law: One giant leap for humankind. En International Law and Power. Perspectives on Legal Order and Justice, editores Kayan Kaikoban y Michael Bohlander,141-162. Leiden: Martinus Nijhoff Publishers.

Mehta, P. (2004). Internally Displaced Persons and the Sardar Sarovar Project: A Case for Rehabilitative Reform in Rural India. Am. U. Int'l L. Rev., 20, 613.

Menéndez, F. M. M. (1999). Derecho internacional público: parte general. Madrid, Trotta.

Rey, A. (2006). Una aproximación a la responsabilidad internacional de las organizaciones internacionales. Valencia: Tirant lo Blanch.

Ryngaert, C., \& Buchanan, H. (2011). Member State responsibility for the acts of international organizations. Utrecht L. Rev., 7, 131

Rosas, D. E. (2007). Batallas en el sistema financiero internacional: Críticas y réplicas contemporáneas en torno al Fondo Monetario Internacional y al Banco Mundial. Boletín Mexicano de Derecho Comparado, (119), 467-529.

Shihata, I. F. (1988). World Bank and Human Rights: An Analysis of the Legal Issues and the Record of Achievements, The. Denv. J. Int'l L. \& Pol'y, 17, 39.

Skogly, S. (2001). Human Rights Obligations of the World Bank and the IMF. Cavendish Publishing.

Suzuki, E., \& Nanwani, S. (2005). Responsibility of international organizations: the accountability mechanisms of multilateral development banks. Mich. J. Int'l L., 27, 177.

Toussaint, E. (2007). Banco Mundial: el golpe de estado permanente: la agenda oculta del Consenso de Washington. Editorial El Viejo Topo. 
World Bank and Organisation for Economic Co-operation and Development (OECD) (2013) Integrating Human Rights into Development: Donor Approaches, Experiences and Challenges, 2nd ed, Washington DC: World Bank.

Wahi, N. (2005). Human rights accountability of the IMF and the World Bank: A critique of existing mechanisms and articulation of a theory of horizontal accountability. UC Davis J. Int'l L. \& Pol'y, 12, 331.

Wouters, J., Brems, E., Smis, S., \& Schmitt, P. (2010). Accountability for Human Rights Violations by International Organisations. Intersentia.

Young, A. I. (2012). Deconstructing International Organization Immunity. Geo. J. Int'l L., $44,311$.

\section{REFERENCIAS ELECTRÓNICAS}

Banco de la República de Colombia (2014). Deuda Externa de Colombia. Recuperado el 15 de Julio de 2014, http://www.banrep.gov.co/

Bank Information Center (2012). Human Rights and the World Bank: Case Studies from IDA Countries. Recuperado el 10 de Julio de 2014, http://www.inclusivedevelopment.net/wpcontent/uploads/2012/02/IDA-Case-Study-Book-FINAL_10-4-13.pdf,

Banco Mundial (2013). Review and Update of the World Bank Safeguard Policies. Recuperado el 10 de Julio de 2014, http://consultations.worldbank.org/Data/hub/files/ consultation-template/review-and-update-world-bank-safeguard-policies/en/documents/ safeguardsreviewstatusreportboardbriefingjuly232013.pdf

Brodnig, G. (2001). The World Bank and Human Rights: Mission Impossible?.Carr Center for Human Rights Policy Working, Paper T-01-05, Recuperado el 5 de mayo de 2014.http:// fletcher. tufts. edu/praxis/xvii/Brodnig. pdf.

Consejo de Europa. Asamblea Parlamentaria (2013). Accountability of international organisations for human rights violations. Recuperado el 6 de julio de 2014,http://assembly.coe.int/nw/ xml/XRef/X2H-Xref-iewPDF.asp?FileID=20310\&lang=en

Gunduz, Canan (2004). Human Rights and Development: The World Bank's Need for a Consistent Approach. Recuperado el 23 de junio de 2014, http://www.lse.ac.uk/ internationalDevelopment/pdf/WP/WP49.pdf 
Human Rights Watch (2013). Abuse Free Development. How the World Bank Should Safeguard Against Human Rights Violations. Recuperado el 12 de junio de 2014. http://www.hrw. org/sites/default/files/reports/worldbank0713_ForUpload.pdf

Koskenniemi, M. (2006). Fragmentation of international law. Difficulties arising from the diversification and expansion of international law. UN. Special report. Recuperado el 20 de julio de 2014. http://untreatyun.org/ilc/documentation/english/a_cn4_I682.pdf

Martin, D. C. (2011). Development's Collateral Damage: The World Bank, involuntary resettlement and human rights. Recuperado el 20 de mayo de 2014, https://www.duo. uio.no/handle/10852/22711

Megnot, T. (2013). The world bank and its development operations in africa: a critical evaluation of its human right accountability mechanisms. Recuperado el 5 de octubre de 2014. http:// etd.aau.edu.et/dspace/handle/123456789/4826

Salomon, M. E. (2007). International economic governance and human rights accountability. Recuperado el 20 de marzo de 2014. http://papers.ssrn.com/sol3/papers.cfm?abstract_ $\mathrm{id}=1013505$

Shams, R (2004). The World Bank as an International Financial Institution. Recuperado el 23 de mayo de 2014, http://papers.ssrn.com/sol3/papers.cfm?abstract_id=588648

Toussaint E (2005). El FMI, el Banco Mundial y el respeto de los derechos humanos. Recuperado el 23 de mayo de 2014, http://www.world- governance.org/article63.html?lang=en. 